\title{
DESIGN, SYNTHESIS AND ANTIMICROBIAL ACTIVITY OF SOME NEW 2,4-DIAMINO PYRIMIDOTHIAZINE DERIVATIVES BY
}

\author{
Helmy M. Sakr
}

FROM

Department of Pharmaceutical Chemistry, Faculty of Pharmacy (Boys), Al-Azhar

University, Nasr City, Cairo, Egypt

\begin{abstract}
The thermal condensation of 2,4-diamino 5-bromo-6-hydroxypyrimidine with 2amino-3-mercaptopropylaniline derivatives (6) and 2-amino-3mercaptopropymethyllaniline derivatives (9) resulted in 2,4-diamino-6-substituted anilinomethlyl 6,7-dihydro-8H-pyrimido[4,5-b][1,4] thiazine derivatives $(\mathbf{1 3}, \mathbf{R}=\mathbf{H})$ and 2,4-diamino-6-substituted methylanilinomethlyl 6,7-dihydro-8-methylpyrimido[4,5-b][1,4] thiazine derivatives $\left(\mathbf{1 3}, \mathbf{R}=\mathbf{C H}_{\mathbf{3}}\right)$ respectively. The synthesized compounds were screened for antimicrobial activity and it was found that the screened compounds have antimicrobial and antifungal activity comparable to chloramphenicol and terbinafine.
\end{abstract}

\section{Introduction}

In the last few years, some of the deaza analogues of tetrahydrofolic acid have been shown to be an important class of potential oncolytic agents which inhibit dihydrofolate reductase, thymidylate synthase or glycinamide ribonucleotide formyl transferase (Gangjee et al 1995 , Gangjee et al 1993, Gangjee et al 1996, Gangjee et al 1998, Gangjee et al 1998 , Gangjee et al 1997, Gangjee et al 1997 , Gangjee et al 1997 , Gangjee et al 1997 , Gangjee et al 1996 , Gangjee et al 1995 )

Tetrahydrofolic acid is an active form of folic acid and plays significant roles as a cofactor for one-carbon transfer in the biosynthesis of aminoacids, nucleosides and nucleotides. The principle goal of this work is to design potent inhibitors of the folate dependent enzymes dihydrofolate reductase (DHFR) that is nonclassical antifolate agents.

\section{Experimental}

All melting points were carried on Gallen Kamp point apparatus and are uncorrected. The infrared spectra were recorded on Brucker-Vector-22F T-IR spectrophotometer using the potassium bromide disc technique. The ${ }^{1} \mathrm{HNMR}$ spectra were recorded on varian-Gemini-300-MHZ spectrophotometer using DMSO-d6 as a solvents and TMS as internal reference. The chemical shift values were recorded in $\delta$ ppm downfield the TMS signal. The mass spectra were recorded on AZH-ph-AR-XO Mass spectrometer. Elemental analyses were performed on $\mathrm{CHN}$ analyzer. All spectral measurements have been performed at the Micro analytical Center, Cairo University, Egypt.

In a previous work (Sakr 2007) we described a new method for the construction of the pyrimido[5,4-b][1,4]thiazine ring system but in this work I decided to synthesize some pyrimido[4,5-b][1,4]thiazine derivatives (13) which have structural similarity to the tetrahydrofolate nucleus (which contain pyrimidopyrazine nucleus), with few exceptions in order to improve some lipophilic antifolate agents. For synthesizing the target compounds (13) the following schemes (1 and $\mathbf{2})$ were adopted. 
Following reported procedures (Sakr 2000), cysteine ethylester hydrochloride was protected as acetonides in good yield, and they are protected with CBZ followed by CBZ deprotection and cleavage of the acetonide with $6 \mathrm{~N} \mathrm{HCl}$ were prepared.

\section{Methylation of 4-substituted anilinomethyl-3-benzyloxycarbonyl-2,2- dimethylthiazolidine (7)}

4-substituted anilinomethyl-3-benzyloxycarbonyl-2,2-dimethylthiazolidine (4) $(0.36 \mathrm{~mol})$ was dissolved in ethanol $(50 \mathrm{ml})$, then methyl iodide $(0.40 \mathrm{~mol})$ was added, the reaction mixture was neutralized with sodium carbonate solution $(12 \%)$ and refluxed for three hours. The solvent was distilled off, the resulted oily product was washed several times with water, and the aqueous mixture was extracted with $\mathrm{CHCl}_{3},(100 \mathrm{X} 4)$. The chloroformic extract was, dried over

anhydrous $\mathrm{MgSO}_{4}$, evaporated to dryness and the resulting residue was purified by column chromatography ad eluted with n-hexane, ethylacetate (20:1) to isolate (7) as oil in $90 \%$ yield.

The structures of these compounds were confirmed by ${ }^{1} \mathrm{HNMR}$ and mass spectra. The ${ }^{1} \mathrm{HNMR}\left(\mathrm{CDCl}_{3}\right)$ spectra of compounds (7) using compounds 7.a and 7.e as representative examples.

Table (1): ${ }^{1} \mathrm{H}$ NMR Spectral data of compounds (7)

\begin{tabular}{|c|c|}
\hline Comp. & multiplicity, protons \\
\hline 7.a & $\begin{array}{l}7.33(5 \mathrm{H}, \mathrm{s}-\mathrm{like}, \text { aromatic protons of CBZ }), 7.14\left(2 \mathrm{H}, \mathrm{brs}, 3^{\prime} \mathrm{H} \text { and } 5^{\prime} \mathrm{H}\right) \text {, } \\
7.06\left(2 \mathrm{H}, \mathrm{d}, 2^{\prime} \mathrm{H} \text { and } 6{ }^{\prime} \mathrm{H}\right), 7.01\left(1 \mathrm{H}, \mathrm{brs}, 4^{\prime} \mathrm{H}\right), 5.3\left(2 \mathrm{H}, \mathrm{s}, \underline{\mathrm{CH}_{2}}-\mathrm{C}_{6} \mathrm{H}_{5}\right) \text {, } \\
4.60(1 \mathrm{H}, \mathrm{s}, 4-\mathrm{H}), 3.52\left(2 \mathrm{H}, \mathrm{m}, \underline{\mathrm{CH}}_{2}-\mathrm{NH}\right), 3.10(1 \mathrm{H}, \mathrm{m}, 5-\mathrm{H}), 2.90(1 \mathrm{H}, \mathrm{d}, \\
5-\mathrm{H}), 1.80\left(6 \mathrm{H}, \mathrm{s}, \mathrm{CH}_{3} \times 2\right), 0.97\left(3 \mathrm{H}, \mathrm{s}, \mathrm{N}-\mathrm{CH}_{3}\right) .\end{array}$ \\
\hline 7.e & $\begin{array}{l}7.40\left(5 \mathrm{H}, \mathrm{s} \text {, aromatic protons of CBZ), } 7.10\left(2 \mathrm{H}, \text { brs, } 3^{\prime} \mathrm{H} \text { and } 5{ }^{\prime} \mathrm{H}\right), 6.65\right. \\
\left(2 \mathrm{H}, \mathrm{d}, 2^{\prime} \mathrm{H} \text { and 6'H), 5.22 }\left(2 \mathrm{H}, \mathrm{s}, \mathrm{CH}_{2}-\mathrm{C}_{6} \mathrm{H}_{5}\right), 4.70(1 \mathrm{H}, \mathrm{s}, 4-\mathrm{H}), 3.97(3 \mathrm{H},\right. \\
\left.\mathrm{s}, \mathrm{O}-\mathrm{CH}_{3}\right), 3.46-3.40\left(2 \mathrm{H}, \mathrm{t}, \underline{\mathrm{CH}}_{2}-\mathrm{NH}\right), 3.20-3.10(1 \mathrm{H}, \mathrm{t}, 5-\mathrm{H}), 1.83(6 \mathrm{H}, \mathrm{s} \text {, } \\
\left.\mathrm{CH}_{3} \times 2\right), 1.10\left(3 \mathrm{H}, \mathrm{s}, \mathrm{N}-\underline{\mathrm{CH}}_{3}\right) .\end{array}$ \\
\hline
\end{tabular}

MS (7.a): (m/z, abound \%): (371, $\left.\mathrm{MH}^{+}, 100 \%\right),(235, \mathrm{M}-\mathrm{CBZ}, 3.10 \%)$, (105, base peak, $92.60 \%)$.

MS (7.e): (m/z, abound \%): (401, $\left.\mathrm{MH}^{+}, 68 \%\right),(136 \mathrm{CBZ}, 100 \%),(105$, base peak, $78.60 \%)$.

\section{CBZ deprotection}

A suspension of (7) $(13.4 \mathrm{mmol})$ and aluminum chloride $(5.3 \mathrm{~g}, 40 \mathrm{mmol})$ in anisole $(300 \mathrm{ml})$ was stirred at room temperature overnight. The reaction was quenched by the addition of water and the aqueous mixture was extracted with ethyl acetate (100 $\mathrm{ml} \times 3$ ). The collected ethyl acetate solution was dried over anhydrous $\mathrm{MgSO}_{4}$ and evaporated to dryness. The residue was subjected to column chromatography and elution with $\mathrm{CHCl}_{3}: \mathrm{Me}_{2} \mathrm{CO}(10: 1)$ provide $(8)$ in $18 \%$ yield as oil.

The structures of these compounds were confirmed by ${ }^{1} \mathrm{HNMR}$ and mass spectra. The ${ }^{1} \mathrm{HNMR}\left(\mathrm{CDCl}_{3}\right)$ spectra of compounds $(\mathbf{8})$ using compounds 8.a and 8.e as representative examples. Comparison of such spectra with that of compound showed the disappearance of the signal at $\delta 7.4$ and $7.3 \mathrm{ppm}$ of the aromatic protons and the signal at $\delta 5.3$ and $5.2 \mathrm{ppm}$ of the benzyl proton. 


\section{Cleavage of the acetonide}

A solution of the $\mathrm{N}$-deprotected thiazolidine $(8)(2.0 \mathrm{mmol})$ in ethanol $(50 \mathrm{ml})$ containing $1 \mathrm{~N} \mathrm{HCl}(5 \mathrm{ml})$ was heated at $60{ }^{\circ} \mathrm{C}$ until the disappearance of the thiazolidine was complete (monitored by TLC; capacity 2 hours). TLC analysis of the reaction showed the occurance of clean reaction. After removal of the solvent under reduced pressure, the resulting residue was used in the next reaction without purification because of its high susceptibility to autoxidation.

The structures of these compounds were confirmed by mass spectra. The mass spectra of such compounds were confirmed by the presence of prominent molecular ion peak.

\section{Bromination of 2,4-diaminopyrimidine}

\section{2,4-diamino-5-pyrimidyl hydrogen sulfate (10)}

Ammonium persulfate $(34.2 \mathrm{gm}$.) in water $(70 \mathrm{ml}$.) was added dropwise to a stirred ice-cold solution of 2,4-diaminopyrimidine $(5.55 \mathrm{gm}, 50 \mathrm{mmol})$ in $3 \mathrm{~N}$ - sodium hydroxide $(220 \mathrm{ml}$.) during 1 hour. After being stirred overnight, the solution was acidified with concentrated hydrochloric acid and the resulted crystalline solid product was filtered off and recrystallized from water to yield (11.8 gm 57.5\%) of 2,4-diamino5-pyrimidyl hydrogen sulfate as pale yellow prismatic needles, m.p. $>300{ }^{\circ} \mathrm{C}$.

\section{2,4-diamino-5- hydroxypyrimidine (11)}

2,4-diamino-5-pyrimidyl hydrogen sulfate $(9.5 \mathrm{gm})$ was heated under reflux in $5 \mathrm{~N}$-hydrochloric acid $(50 \mathrm{ml}$.) during 30 minutes. After the solution being cooled the formed crystalline product was filtered off and recrystallized from water to yield (6.0 gm, $81 \%$ ) in needles m.p. $>300{ }^{\circ} \mathrm{C}$.

\section{Pyrimido[4,5-b][1,4]thiazine derivatives. (13)}

$N$-Bromosuccinamide $(0.195 \mathrm{gm} ., 1.1 \mathrm{mmol})$ was added to a suspension of (11) $(1.0 \mathrm{mmol})$ in ethanol $(10 \mathrm{ml})$ and the mixture was stirred at room temperature for 1 hour. Compound (6 or 9) $(2.0 \mathrm{mmol})$ was added and the reaction mixture was refluxed for 36-40 hours, after removal of the solvent under reduced pressure the resulting solid was crystallized from ethanol to give compounds (13) in $45 \%$ yield.

Table (2): The physical data and elemental analysis of 2,4-diamino-6-substituted anilinomethyl-6,7-dihydro-3H,5H-pyrimido[4,5-b][1,4]thiazine derivatives. (13)

\begin{tabular}{|c|c|c|c|c|c|c|c|c|}
\hline \multirow{2}{*}{$\begin{array}{l}\text { Comp. } \\
\text { No. }\end{array}$} & \multirow[t]{2}{*}{$\mathbf{R}$} & \multirow[t]{2}{*}{$\mathbf{R}^{\prime}$} & \multirow[t]{2}{*}{ m.p. ${ }^{\circ} \mathrm{C}$} & \multirow[t]{2}{*}{ Molecular Formula } & \multirow{2}{*}{$\begin{array}{l}\text { Mol. } \\
\text { Weight }\end{array}$} & \multicolumn{3}{|c|}{ Elemental analysis } \\
\hline & & & & & & $\mathbf{C}$ & $\mathbf{H}$ & $\mathbf{N}$ \\
\hline 13.1 & $\mathrm{H}$ & $\mathrm{H}$ & $225-227$ & $\mathrm{C}_{13} \mathrm{H}_{16} \mathrm{SN}_{6}$ & 288 & $\begin{array}{l}54.16 \\
54.16\end{array}$ & $\begin{array}{l}5.55 \\
5.57\end{array}$ & $\begin{array}{l}29.16 \\
29.17\end{array}$ \\
\hline 13.2 & $\mathrm{H}$ & $2-\mathrm{Cl}$ & $240-242$ & $\mathrm{C}_{13} \mathrm{H}_{15} \mathrm{SN}_{6} \mathrm{Cl}$ & 322 & $\begin{array}{l}48.44 \\
48.46\end{array}$ & $\begin{array}{l}4.65 \\
4.65\end{array}$ & $\begin{array}{l}26.08 \\
26.08\end{array}$ \\
\hline 13.3 & $\mathrm{H}$ & $3-\mathrm{Cl}$ & 194-197 & $\mathrm{C}_{13} \mathrm{H}_{15} \mathrm{SN}_{6} \mathrm{Cl}$ & 322 & $\begin{array}{l}48.44 \\
48.48\end{array}$ & $\begin{array}{l}4.65 \\
4.67\end{array}$ & $\begin{array}{l}26.08 \\
26.10\end{array}$ \\
\hline 13.4 & $\mathrm{H}$ & $4-\mathrm{Cl}$ & $223-225$ & $\mathrm{C}_{13} \mathrm{H}_{15} \mathrm{SN}_{6} \mathrm{Cl}$ & 322 & $\begin{array}{l}48.44 \\
48.46\end{array}$ & $\begin{array}{l}4.65 \\
4.66\end{array}$ & $\begin{array}{l}26.08 \\
26.11\end{array}$ \\
\hline 13.5 & $\mathrm{H}$ & $2-\mathrm{OCH}_{3}$ & $208-210$ & $\mathrm{C}_{14} \mathrm{H}_{18} \mathrm{SN}_{6} \mathrm{O}$ & 318 & $\begin{array}{l}52.83 \\
52.86\end{array}$ & $\begin{array}{l}5.66 \\
5.67\end{array}$ & $\begin{array}{l}26.41 \\
26.42\end{array}$ \\
\hline 13.6 & $\mathrm{H}$ & $3-\mathrm{OCH}_{3}$ & $198-200$ & $\mathrm{C}_{14} \mathrm{H}_{18} \mathrm{SN}_{6} \mathrm{O}$ & 318 & $\begin{array}{l}52.83 \\
52.85\end{array}$ & $\begin{array}{l}5.66 \\
5.66\end{array}$ & $\begin{array}{l}26.41 \\
26.41\end{array}$ \\
\hline 13.7 & $\mathrm{H}$ & $4-\mathrm{OCH}_{3}$ & $201-203$ & $\mathrm{C}_{14} \mathrm{H}_{18} \mathrm{SN}_{6} \mathrm{O}$ & 318 & $\begin{array}{l}52.83 \\
52.87\end{array}$ & $\begin{array}{l}5.66 \\
5.68\end{array}$ & $\begin{array}{l}26.41 \\
26.43\end{array}$ \\
\hline 13.8 & $\mathrm{H}$ & $2,4-\mathrm{Cl}_{2}$ & $248-250$ & $\mathrm{C}_{13} \mathrm{H}_{14} \mathrm{SN}_{6} \mathrm{Cl}_{2}$ & 356 & $\begin{array}{l}43.82 \\
43.85\end{array}$ & $\begin{array}{l}3.93 \\
3.97\end{array}$ & $\begin{array}{l}23.59 \\
23.62\end{array}$ \\
\hline 13.9 & $\mathrm{H}$ & $3,5-\mathrm{Cl}_{2}$ & $266-268$ & $\mathrm{C}_{13} \mathrm{H}_{14} \mathrm{SN}_{6} \mathrm{Cl}_{2}$ & 356 & $\begin{array}{l}43.82 \\
43.84\end{array}$ & $\begin{array}{l}3.93 \\
3.93\end{array}$ & $\begin{array}{l}23.59 \\
23.61\end{array}$ \\
\hline 13.10 & $\mathrm{H}$ & $3,4,5-\left(\mathrm{OCH}_{3}\right)_{3}$ & $227-229$ & $\mathrm{C}_{16} \mathrm{H}_{22} \mathrm{SN}_{6} \mathrm{O}_{3}$ & 378 & $\begin{array}{l}50.79 \\
50.82\end{array}$ & $\begin{array}{l}5.82 \\
5.84\end{array}$ & $\begin{array}{l}22.22 \\
22.24\end{array}$ \\
\hline
\end{tabular}


Table (3): The physical data and elemental analysis of 2,4-diamino-6-substituted methylanilinomethyl-6,7-dihydro-3H,5H-pyrimido $[4,5-b][1,4]$ thiazine derivatives. (13)

\begin{tabular}{|c|c|c|c|c|c|c|c|c|}
\hline \multirow{2}{*}{$\begin{array}{l}\text { Comp. } \\
\text { NO. }\end{array}$} & \multirow[t]{2}{*}{$\mathbf{R}$} & \multirow[t]{2}{*}{$\mathrm{R}^{\prime}$} & \multirow[t]{2}{*}{ m.p. ${ }^{\circ} \mathrm{C}$} & \multirow{2}{*}{$\begin{array}{l}\text { Molecular } \\
\text { Formula }\end{array}$} & \multirow{2}{*}{$\begin{array}{l}\text { Mol. } \\
\text { Weight }\end{array}$} & \multicolumn{3}{|c|}{ Elemental analysis } \\
\hline & & & & & & $\mathbf{C}$ & $\mathbf{H}$ & $\mathbf{N}$ \\
\hline 13.11 & $\mathrm{CH}_{3}$ & $\mathrm{H}$ & $167-169$ & $\mathrm{C}_{14} \mathrm{H}_{18} \mathrm{SN}_{6}$ & 302 & $\begin{array}{l}55.63 \\
55.67\end{array}$ & $\begin{array}{l}5.96 \\
5.98\end{array}$ & $\begin{array}{l}27.81 \\
27,84\end{array}$ \\
\hline 13.12 & $\mathrm{CH}_{3}$ & $2-\mathrm{Cl}$ & 197-199 & $\mathrm{C}_{14} \mathrm{H}_{17} \mathrm{SN}_{6} \mathrm{Cl}$ & 336 & $\begin{array}{l}50.00 \\
50.03\end{array}$ & $\begin{array}{l}5.06 \\
5.10\end{array}$ & $\begin{array}{l}25.00 \\
25,02\end{array}$ \\
\hline 13.13 & $\mathrm{CH}_{3}$ & $3-\mathrm{Cl}$ & $179-181$ & $\mathrm{C}_{14} \mathrm{H}_{17} \mathrm{SN}_{6} \mathrm{Cl}$ & 336 & $\begin{array}{l}50.00 \\
50.05\end{array}$ & $\begin{array}{l}5.06 \\
5.07\end{array}$ & $\begin{array}{l}25.00 \\
25.03\end{array}$ \\
\hline 13.14 & $\mathrm{CH}_{3}$ & $4-\mathrm{Cl}$ & $195-197$ & $\mathrm{C}_{14} \mathrm{H}_{17} \mathrm{SN}_{6} \mathrm{Cl}$ & 336 & $\begin{array}{l}50.00 \\
50.04\end{array}$ & $\begin{array}{l}5.06 \\
5.08\end{array}$ & $\begin{array}{l}25.00 \\
25.05\end{array}$ \\
\hline 13.15 & $\mathrm{CH}_{3}$ & $2-\mathrm{OCH}_{3}$ & $203-205$ & $\mathrm{C}_{15} \mathrm{H}_{20} \mathrm{SN}_{6} \mathrm{O}$ & 332 & $\begin{array}{l}54.21 \\
54.23\end{array}$ & $\begin{array}{l}6.02 \\
6.04\end{array}$ & $\begin{array}{l}25.30 \\
25.33\end{array}$ \\
\hline 13.16 & $\mathrm{CH}_{3}$ & $3-\mathrm{OCH}_{3}$ & $187-189$ & $\mathrm{C}_{15} \mathrm{H}_{20} \mathrm{SN}_{6} \mathrm{O}$ & 332 & $\begin{array}{l}54.21 \\
54.24\end{array}$ & $\begin{array}{l}6.02 \\
6.02\end{array}$ & $\begin{array}{l}25.30 \\
25.34\end{array}$ \\
\hline 13.17 & $\mathrm{CH}_{3}$ & $4-\mathrm{OCH}_{3}$ & 196-198 & $\mathrm{C}_{15} \mathrm{H}_{20} \mathrm{SN}_{6} \mathrm{O}$ & 332 & $\begin{array}{l}54.21 \\
54.26\end{array}$ & $\begin{array}{l}6.02 \\
6.07\end{array}$ & $\begin{array}{l}25.30 \\
25.31\end{array}$ \\
\hline 13.18 & $\mathrm{CH}_{3}$ & $2,4-\mathrm{Cl}_{2}$ & $223-225$ & $\mathrm{C}_{14} \mathrm{H}_{16} \mathrm{SN}_{6} \mathrm{Cl}_{2}$ & 370 & $\begin{array}{l}45.40 \\
45.43\end{array}$ & $\begin{array}{l}4.32 \\
4.36\end{array}$ & $\begin{array}{l}22.70 \\
22.72\end{array}$ \\
\hline 13.19 & $\mathrm{CH}_{3}$ & $3,5-\mathrm{Cl}_{2}$ & $231-233$ & $\mathrm{C}_{14} \mathrm{H}_{16} \mathrm{SN}_{6} \mathrm{Cl}_{2}$ & 370 & $\begin{array}{l}45.40 \\
45.44\end{array}$ & $\begin{array}{l}4.32 \\
4.34\end{array}$ & $\begin{array}{l}22.70 \\
22.71\end{array}$ \\
\hline 13.20 & $\mathrm{CH}_{3}$ & $\begin{array}{l}3,4,5- \\
\left(\mathrm{OCH}_{3}\right)_{3}\end{array}$ & $219-221$ & $\mathrm{C}_{17} \mathrm{H}_{24} \mathrm{SN}_{6} \mathrm{O}_{3}$ & 392 & $\begin{array}{l}52.04 \\
52.08\end{array}$ & $\begin{array}{l}6.12 \\
6.14\end{array}$ & $\begin{array}{l}21.42 \\
21.45\end{array}$ \\
\hline
\end{tabular}


Table (4): ${ }^{1} \mathrm{H}$ NMR Spectral data of compounds (13)

\begin{tabular}{|c|c|}
\hline Comp. & $\delta$, multiplicity, protons \\
\hline 13.1 & $\begin{array}{l}7.74\left(2 \mathrm{H}, \text { brs, } 4-\mathrm{NH}_{2}, \text { exchange }\right), 7.23(2 \mathrm{H}, \mathrm{dd}, 3 \mathrm{H} \text { and } 5 \mathrm{H}), 6.99\left(2 \mathrm{H}, \text { brs, } 2-\mathrm{NH}_{2} \text {, }\right. \\
\text { exchange }), 6.58(2 \mathrm{H}, \mathrm{d}, 2 \mathrm{H} \text { and } 6 \mathrm{H}), 6.77\left(1 \mathrm{H}, \mathrm{s}, 4^{\prime} \mathrm{H}\right), 5.28(1 \mathrm{H}, \mathrm{brs}, 4-\mathrm{NH}), 4.0(1 \mathrm{H} \text {, } \\
\text { brs, 5- NH, exchange }), 3.41-3.38\left(2 \mathrm{H}, \mathrm{d}, 9-\mathrm{CH}_{2}\right), 3.25-3.04\left(2 \mathrm{H}, \mathrm{m}, 7-\mathrm{CH}_{2}\right), 3.13-2.93 \\
(1 \mathrm{H}, \mathrm{m}, 6-\mathrm{CH}) .\end{array}$ \\
\hline 13.2 & 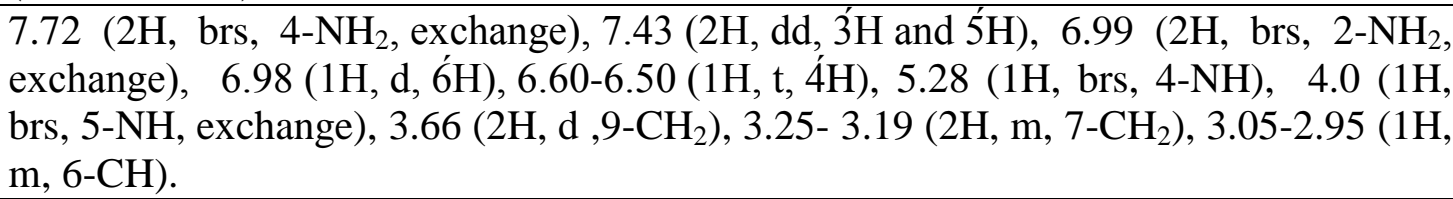 \\
\hline 13.8 & 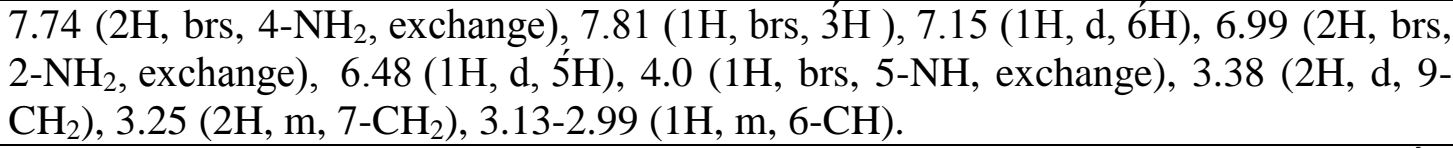 \\
\hline 13.10 & $\begin{array}{l}7.74\left(2 \mathrm{H}, \text { brs, } 4-\mathrm{NH}_{2} \text {, exchange }\right), 6.99\left(2 \mathrm{H}, \text { brs, } 2-\mathrm{NH}_{2} \text {, exchange }\right), 5.60(1 \mathrm{H}, \text { brs, } 2 \mathrm{H} \\
\text { and } 6 \dot{\mathrm{H}}), 6.99\left(2 \mathrm{H}, \mathrm{brs}, 4-\mathrm{NH}_{2} \text {, exchange }\right), 5.28(1 \mathrm{H}, \mathrm{brs}, 4-\mathrm{NH}), 4.0(1 \mathrm{H}, \mathrm{brs}, 5-\mathrm{NH} \text {, } \\
\text { exchange }), 3.83\left(9 \mathrm{H}, \mathrm{s}-\mathrm{like}, \mathrm{OCH}_{3} \times 3\right), 3.38\left(2 \mathrm{H}, \mathrm{d}, 9-\mathrm{CH}_{2}\right), 3.25-3.18(2 \mathrm{H}, \mathrm{m}, 7- \\
\left.\mathrm{CH}_{2}\right), 3.13-2.90(1 \mathrm{H}, \mathrm{m}, 6-\mathrm{CH}) .\end{array}$ \\
\hline 13.11 & $\begin{array}{l}7.74\left(2 \mathrm{H}, \text { brs, 4-NH } \mathrm{NH}_{2} \text { exch. }\right), 7.27(2 \mathrm{H}, \mathrm{dd}, 3 \mathrm{H} \text { and } 5 \mathrm{H}), 6.99\left(2 \mathrm{H}, \text { brs, 2- } \mathrm{NH}_{2} \text {, }\right. \\
\text { exchange }), 6.94(2 \mathrm{H}, \mathrm{d}, 2 \mathrm{H} \text { and } 6 \mathrm{H}), 6.79\left(1 \mathrm{H}, \mathrm{s}, 4^{\prime} \mathrm{H}\right), 5.28(1 \mathrm{H}, \mathrm{brs}, 4-\mathrm{NH}), 3.67- \\
3.60\left(2 \mathrm{H}, \mathrm{d}, 9-\mathrm{CH}_{2}\right), 3.25-3.19\left(2 \mathrm{H}, \mathrm{m}, 7-\mathrm{CH}_{2}\right), 3.13-2.90(1 \mathrm{H}, \mathrm{m}, 6-\mathrm{CH}), 2.75(3 \mathrm{H} \text {, } \\
\left.\text { brs } \mathrm{N}-\mathrm{CH}_{3}\right)\end{array}$ \\
\hline 13.12 & 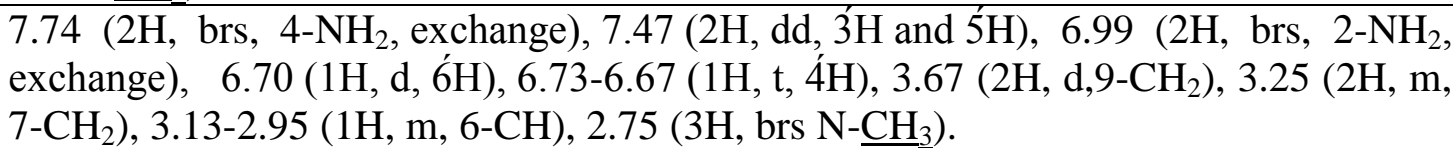 \\
\hline 13.18 & $\begin{array}{l}7.74(2 \mathrm{H}, \text { brs, 4-NH} \\
\text { 2- exchange }), 7.85(1 \mathrm{H}, \text { brs, } 3 \mathrm{H}), 7.19(1 \mathrm{H}, \mathrm{d}, 5 \mathrm{H}), 6.99(2 \mathrm{H}, \text { brs, } \\
2.99(1 \mathrm{H}, \mathrm{m}, 6-\mathrm{CH}), 2.75\left(3 \mathrm{H}, \text { brs } \mathrm{N}-\underline{\mathrm{CH}}_{3}\right) .\end{array}$ \\
\hline 13.20 & 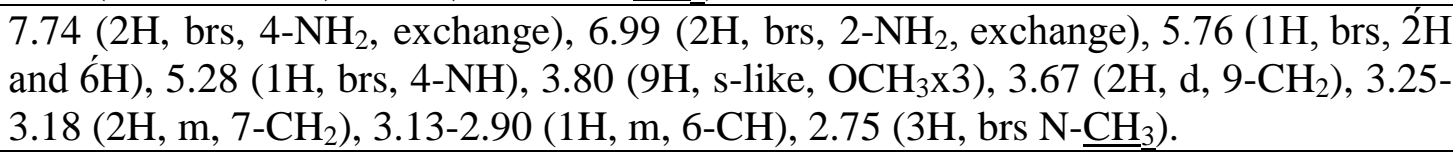 \\
\hline
\end{tabular}

MS (13.4): (m/z, abound \%): 323/325, $\left.\mathrm{MH}^{+}, 100 / 37 \%\right),\left(183 / 185, \quad \mathrm{M}-\mathrm{C}_{7} \mathrm{H}_{7} \mathrm{NCl}\right.$, 93/31\%).

IR (13.4): $\left(\mathrm{KBr}, \mathrm{cm}^{-1}\right) 3418$ (s, NH), 3039 (s,C-H, aromatic), 2873 (s, C-H, alkane), 1514 (s, $\mathrm{C}=\mathrm{C}$, aromatic).

MS (13.7): (m/z, abound \%): (319, $\left.\mathrm{MH}^{+}, 78.40 \%\right),\left(182, \mathrm{M}_{-} \mathrm{C}_{8} \mathrm{H}_{10} \mathrm{NO}, 23.10 \%\right)$.

IR (13.7): $\left(\mathrm{KBr}, \mathrm{cm}^{-1}\right) 3472$ (s, NH), 3037 (s,C-H, aromatic), 2903 (s, C-H, alkane), 1542 ( $\mathrm{s}, \mathrm{C}=\mathrm{C}$, aromatic).

IR (13.11): (KBr, cm $\left.{ }^{-1}\right) 3507$ (s, NH), 3042 (s,C-H, aromatic), 2893 (s, C-H, alkane), 1574 (s, C=C, aromatic).

MS (13.11): (m/z, abound \%): $\left(303, \mathrm{MH}^{+}, 100 \%\right)$

IR (13.20): $\left(\mathrm{KBr}, \mathrm{cm}^{-1}\right) 3497$ (s, NH), 3058 (s,C-H, aromatic), 2912 (s, C-H,alkane), $1570(\mathrm{~s}, \mathrm{C}=\mathrm{C}$, aromatic).

MS (13.20): (m/z, abound \%): $\left(393, \mathrm{MH}^{+}, 100 \%\right)$ 


\section{Antibacterial Testing}

The antimicrobial activity of the newly synthesized compounds was tested by measuring the inhibitory effect of such compounds on the culture growth of certain pathogenic bacteria and fungi using agar diffusion method.( Hewth et al 1989 ) The test as achieved by the regional center for Mycology and Biotechnology, Al-Azhar university, Cairo, Egypt.

\section{Material and Methods}

The microorganism used in this study include Aspergillus fumigatus, Penicillium italicum, Syncephalasteum racemosum, Candida albicans, Staphylococcus aureus, Pseudomonas aeraginosa, Bacillus subtilis, and Escherichia coli were used against the test compound and obtained from the regional center, Chloramphenicol was used as antibacterial standard and Terbinafin was used as antifungal standard.

\section{Preparation of bacterial suspensions}

Suspensions of the above mention microorganisms were prepared by inoculating fresh stock cultures into separate broth tubes, each containing $7 \mathrm{ml}$ of nutrient broth (peptone, $0.3 \%$ ), (beef extract $0.3 \%$ ). The inoculated tubes were incubated at $37^{\circ} \mathrm{C}$ for 24 hours.

\section{Preparation of test compounds solutions}

Solutions of test compounds were prepared by dissolving of $0.5 \mathrm{~g}$ of each compound in $10 \mathrm{ml}$ of dimethylformamide. Solutions of chloramphenicol and terbinafin were prepared in the same manner.

The antimicrobial activity of the different compounds has been presented in term of diameter of inhibitions zones. Moreover, the data as well as the grading scores of antimicrobial activity are shown in Table (5). 
Table (5): Antimicrobial testing of the synthesized compounds

\begin{tabular}{|c|c|c|c|c|c|c|c|c|}
\hline \multirow[b]{2}{*}{ Comp. No. } & \multicolumn{8}{|c|}{ Microorganism (inhibition zone) (mm) } \\
\hline & $\begin{array}{c}\text { Asp. } \\
\text { fumigat } \\
\text { es }\end{array}$ & $\begin{array}{l}\text { Penicilli } \\
\text { um } \\
\text { italicum }\end{array}$ & $\begin{array}{l}\text { Synceph. } \\
\text { racemosum }\end{array}$ & $\begin{array}{l}\text { Candida } \\
\text { albicans }\end{array}$ & $\begin{array}{l}\text { Staph. } \\
\text { aureus }\end{array}$ & $\begin{array}{c}P . \\
\text { aerugin } \\
\text { osa }\end{array}$ & $\begin{array}{c}\text { B. } \\
\text { subtiles }\end{array}$ & $\begin{array}{l}\text { E. } \\
\text { coli }\end{array}$ \\
\hline 11.1 & $14 \mathrm{~mm}$ & $15 \mathrm{~mm}$ & $16 \mathrm{~mm}$ & $8 \mathrm{~mm}$ & $14 \mathrm{~mm}$ & $10 \mathrm{~mm}$ & $10 \mathrm{~mm}$ & $12 \mathrm{~mm}$ \\
\hline 11.2 & $15 \mathrm{~mm}$ & $16 \mathrm{~mm}$ & $16 \mathrm{~mm}$ & $10 \mathrm{~mm}$ & $12 \mathrm{~mm}$ & $14 \mathrm{~mm}$ & $12 \mathrm{~mm}$ & $14 \mathrm{~mm}$ \\
\hline 11.3 & $15 \mathrm{~mm}$ & $16 \mathrm{~mm}$ & $16 \mathrm{~mm}$ & $6 \mathrm{~mm}$ & $14 \mathrm{~mm}$ & $14 \mathrm{~mm}$ & $12 \mathrm{~mm}$ & $14 \mathrm{~mm}$ \\
\hline 11.4 & $14 \mathrm{~mm}$ & $14 \mathrm{~mm}$ & $14 \mathrm{~mm}$ & $6 \mathrm{~mm}$ & $12 \mathrm{~mm}$ & $12 \mathrm{~mm}$ & $10 \mathrm{~mm}$ & $14 \mathrm{~mm}$ \\
\hline 11.5 & $16 \mathrm{~mm}$ & $18 \mathrm{~mm}$ & $14 \mathrm{~mm}$ & $6 \mathrm{~mm}$ & $12 \mathrm{~mm}$ & $13 \mathrm{~mm}$ & $12 \mathrm{~mm}$ & $12 \mathrm{~mm}$ \\
\hline 11.6 & $14 \mathrm{~mm}$ & $16 \mathrm{~mm}$ & $14 \mathrm{~mm}$ & $8 \mathrm{~mm}$ & $14 \mathrm{~mm}$ & $14 \mathrm{~mm}$ & $10 \mathrm{~mm}$ & $14 \mathrm{~mm}$ \\
\hline 11.7 & $12 \mathrm{~mm}$ & $14 \mathrm{~mm}$ & $15 \mathrm{~mm}$ & $8 \mathrm{~mm}$ & $14 \mathrm{~mm}$ & $12 \mathrm{~mm}$ & $12 \mathrm{~mm}$ & $14 \mathrm{~mm}$ \\
\hline 11.8 & $12 \mathrm{~mm}$ & $16 \mathrm{~mm}$ & $16 \mathrm{~mm}$ & $6 \mathrm{~mm}$ & - & $10 \mathrm{~mm}$ & $10 \mathrm{~mm}$ & $10 \mathrm{~mm}$ \\
\hline 11.9 & $12 \mathrm{~mm}$ & $16 \mathrm{~mm}$ & $14 \mathrm{~mm}$ & $6 \mathrm{~mm}$ & - & $8 \mathrm{~mm}$ & $10 \mathrm{~mm}$ & $10 \mathrm{~mm}$ \\
\hline 11.10 & $15 \mathrm{~mm}$ & $18 \mathrm{~mm}$ & $16 \mathrm{~mm}$ & $10 \mathrm{~mm}$ & $14 \mathrm{~mm}$ & $14 \mathrm{~mm}$ & $14 \mathrm{~mm}$ & $15 \mathrm{~mm}$ \\
\hline 11.11 & $12 \mathrm{~mm}$ & $12 \mathrm{~mm}$ & $12 \mathrm{~mm}$ & $6 \mathrm{~mm}$ & $12 \mathrm{~mm}$ & $8 \mathrm{~mm}$ & $12 \mathrm{~mm}$ & $14 \mathrm{~mm}$ \\
\hline 11.12 & $12 \mathrm{~mm}$ & $12 \mathrm{~mm}$ & $12 \mathrm{~mm}$ & $5 \mathrm{~mm}$ & $12 \mathrm{~mm}$ & $10 \mathrm{~mm}$ & $14 \mathrm{~mm}$ & $14 \mathrm{~mm}$ \\
\hline 11.13 & $12 \mathrm{~mm}$ & $15 \mathrm{~mm}$ & $16 \mathrm{~mm}$ & $6 \mathrm{~mm}$ & $12 \mathrm{~mm}$ & $12 \mathrm{~mm}$ & $10 \mathrm{~mm}$ & $12 \mathrm{~mm}$ \\
\hline 11.14 & $14 \mathrm{~mm}$ & $12 \mathrm{~mm}$ & $16 \mathrm{~mm}$ & $6 \mathrm{~mm}$ & $14 \mathrm{~mm}$ & $14 \mathrm{~mm}$ & $10 \mathrm{~mm}$ & $14 \mathrm{~mm}$ \\
\hline 11.15 & $12 \mathrm{~mm}$ & $14 \mathrm{~mm}$ & - & - & - & $12 \mathrm{~mm}$ & $12 \mathrm{~mm}$ & $10 \mathrm{~mm}$ \\
\hline 11.16 & $10 \mathrm{~mm}$ & $14 \mathrm{~mm}$ & - & - & - & $12 \mathrm{~mm}$ & $10 \mathrm{~mm}$ & $12 \mathrm{~mm}$ \\
\hline 11.17 & $10 \mathrm{~mm}$ & $16 \mathrm{~mm}$ & $14 \mathrm{~mm}$ & $8 \mathrm{~mm}$ & $12 \mathrm{~mm}$ & $12 \mathrm{~mm}$ & $14 \mathrm{~mm}$ & $12 \mathrm{~mm}$ \\
\hline 11.18 & $10 \mathrm{~mm}$ & $14 \mathrm{~mm}$ & $12 \mathrm{~mm}$ & $6 \mathrm{~mm}$ & $12 \mathrm{~mm}$ & $14 \mathrm{~mm}$ & $12 \mathrm{~mm}$ & $10 \mathrm{~mm}$ \\
\hline 11.19 & $10 \mathrm{~mm}$ & $16 \mathrm{~mm}$ & $14 \mathrm{~mm}$ & $6 \mathrm{~mm}$ & $14 \mathrm{~mm}$ & $10 \mathrm{~mm}$ & $14 \mathrm{~mm}$ & $10 \mathrm{~mm}$ \\
\hline 11.20 & $16 \mathrm{~mm}$ & $18 \mathrm{~mm}$ & $16 \mathrm{~mm}$ & $8 \mathrm{~mm}$ & $16 \mathrm{~mm}$ & $15 \mathrm{~mm}$ & $16 \mathrm{~mm}$ & $15 \mathrm{~mm}$ \\
\hline $\begin{array}{c}\text { Chloramphenic } \\
\text { ol }\end{array}$ & $20 \mathrm{~mm}$ & $20 \mathrm{~mm}$ & $20 \mathrm{~mm}$ & - & $20 \mathrm{~mm}$ & $20 \mathrm{~mm}$ & $20 \mathrm{~mm}$ & $15 \mathrm{~mm}$ \\
\hline Terbinafine & - & - & - & $10 \mathrm{~mm}$ & - & - & - & - \\
\hline
\end{tabular}

\section{Result and discussion.}

The reported method (Sakr et al 2007) for the synthesis of 4-Substituted anilinomethyl-3-benzyloxycarbonyl-2,2-dimethylthiazolidine (4) derivatives was modified which is prepared through the condensation of aniline derivatives with the aldehyde (3) followed by reduction of the amide group by using $\mathrm{LiAlH}_{4}$ in dry ether or dry tetrahydrofuran in good yield and the resulted product was methylated with methyliodide in ethanol to yield the N-methyl product (7) the structure of compounds (7) were confirmed by spectral data including ${ }^{1} \mathrm{HNMR}$ and mass spectra. The benzyl carbamates protecting group was removed by using aluminum trichloride in the presence of anisole at $0{ }^{\circ} \mathrm{C}$ or by using hydrogen bromide in glacial acetic acid at room 
temperature to yield compounds (5 and 8) in good yield and the structure of the $\mathrm{N}$ methylated product was confirmed by ${ }^{1} \mathrm{HNMR}$ which is characterized by the disappearance of the aromatic proton of the benzyl group. The aminothiol derivatives (6 and 9) were prepared by cleavage of the acetonide by stirring with ethanolic dilute $\mathrm{HCl}$ at $60{ }^{\circ} \mathrm{C}$ in good yield and its used in the next step without purification due to oxidation. The bromohydroxypyrimidine (12) is an essential intermediate for thermal condensation with aminothiol derivatives (6 and 9) in order to get the final compounds. In the present work 2,4-diamino-6-bromo-5-hydroxypyrimidine (12) was prepared through the reaction of 2,4-diaminopyrimidine with alkaline sodium persulphate to form 2,4diamino-5-pyrimidyl hydrogen sulphate which was hydrolyzed to 2,4diamino-5hydroxypyrimidine (11) by refluxing in $5 \mathrm{~N} \mathrm{HCl}$ during 30 minutes. 2,4-diamino-5hydroxypyrimidine was treated with $N$-bromosuccinamide (NBS) in order to get 2,4diamino-6-bromo-5-hydroxypyrimidine (12). The aminothiol derivatives (6 and 9) and 2,4-diamino-6-bromo-5-hydroxypyrimidine (12) were refluxed together in ethanol to get the final product (13) in 55\% yield. The initially formed 6-bromo-5hydroxypyrimidine is trapped by ethanol to give the dihydropyrimidine intermediate. The dihydropyrimidine could react with aminothiol derivatives under thermal conditions to produce the pyrimido[4,5-b][1,4]thiazine (13) in the presence of acid catalyst. The structures of the target compounds were confirmed by elemental and spectral data. The ${ }^{1}$ HNMR spectra of compounds $\mathbf{1 3}$ are characterized by the presence of broad singlet of one proton at a range of $\delta 10.86-10.83 \mathrm{ppm}$ due to $3-\mathrm{NH}$. The aromatic protons appeared at the region between $\delta 7.08-5.82 \mathrm{ppm}$ depending on the position of the subsistent and the derivatives on the phenyl ring. There is a broad singlet of two protons at a range of $\delta 6.10-5.82 \mathrm{ppm}$ due to amino group at C-2, broad singlet of one proton at $\delta 5.86$ due to $5-\mathrm{NH}$, a multiplet of one proton at $\delta 3.50-3.33 \mathrm{ppm}$ due to the 6- $\mathrm{CH}$ proton, while the methylene protons at $\mathrm{C}-9$ were appeared as doublet of two protons between $\delta 3.19-3.05 \mathrm{ppm}$. The methylene protons at C-7 are characterized by the presence of two multiplets of two protons at $\delta 2.90 \mathrm{ppm}$. The ${ }^{1} \mathrm{HNMR}$ of compounds $\mathbf{1 3 . 2}$ are characterized by the presence of peaks at the region between $\delta$ 7.32-6.58 ppm due to the aromatic protons and two broad singlets of one proton at $\delta$ 7.74 and $6.99 \mathrm{ppm}$ due to $4-\mathrm{NH}_{2}$ and $2-\mathrm{NH}_{2}$ respectively. The 5-NH appears as broad singlet at $\delta 5.28 \mathrm{ppm}$. The difference between compounds 20.1-10 and compounds 20 . 11-20 is the disappearance of broad signal at $\delta 4.00$ due to $\mathrm{NH}$ and the appearance of broad signal at $\delta 2.75$ due to $\mathrm{N}-\mathrm{CH}_{3}$. The EI-Mass spectrum of compounds $(\mathbf{1 3 . 2} \&$ 13.3) showed prominent $\mathrm{MH}^{+}$and other peaks due to fragmentation of the base peak. All the synthesized compounds have antibacterial activity compared to chloramphenicol as shown in table (5). 


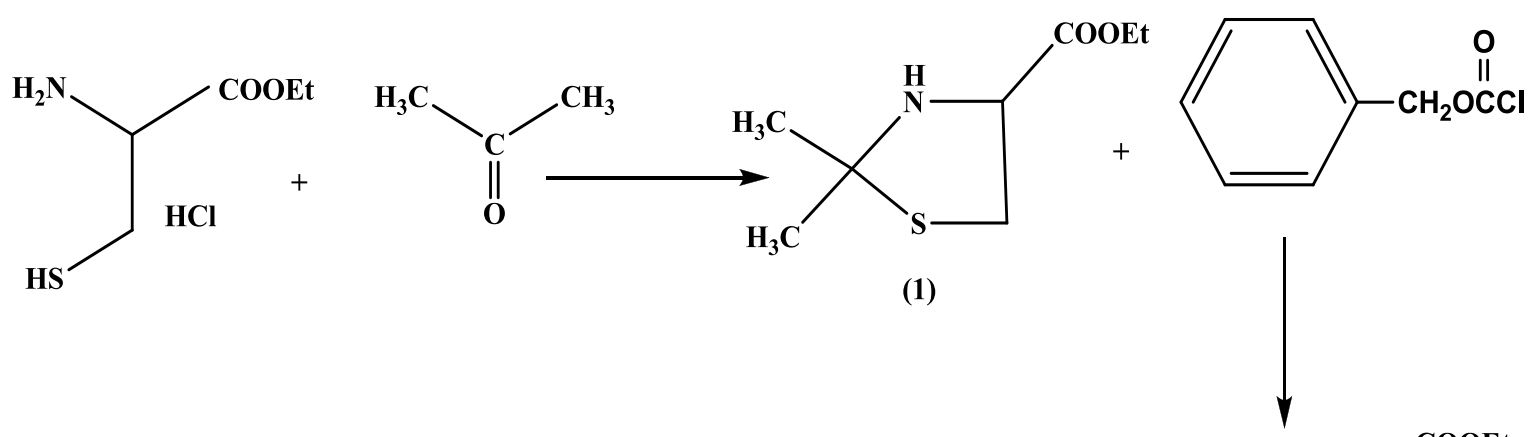

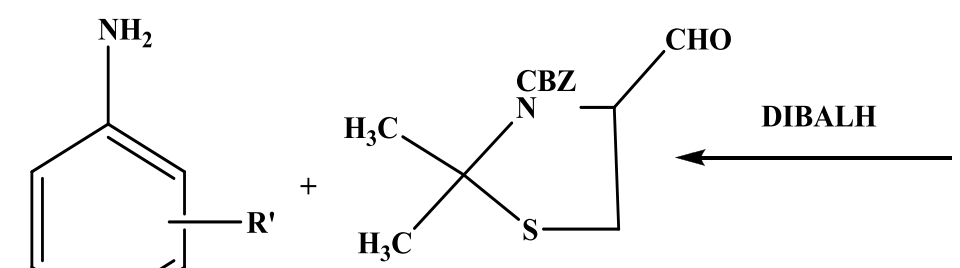

(3)<smiles></smiles>

(2)

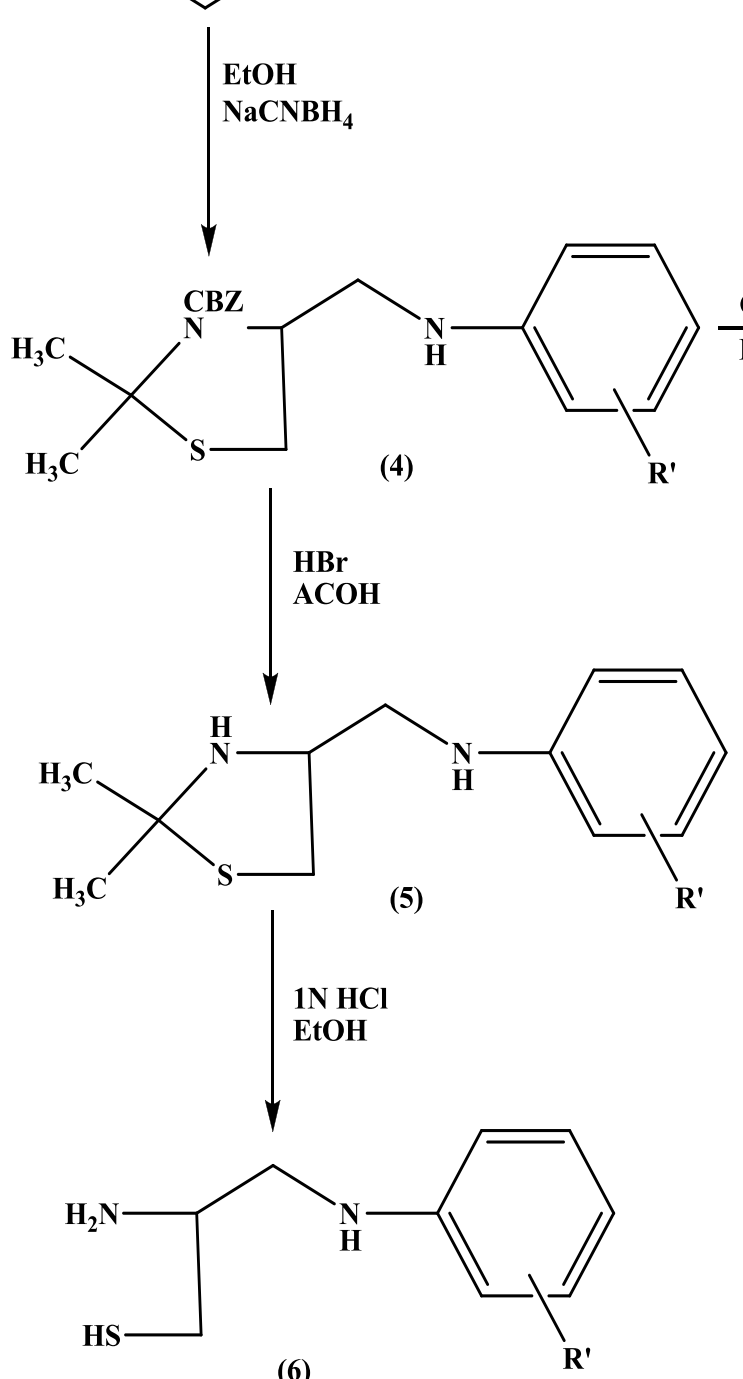

(6)<smiles>[R]c1cccc(N(C)CC2CSC(C)(C(=O)O)[N]2)c1</smiles>

$\mathrm{R}^{\prime}=\mathrm{H}, \mathbf{2}-\mathrm{Cl}, \mathbf{3 - C l}, \mathbf{4 - C l}, \mathbf{2}-\mathrm{OCH}_{3}, \mathbf{3 -}-\mathrm{OCH}_{3}$,

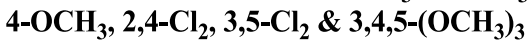




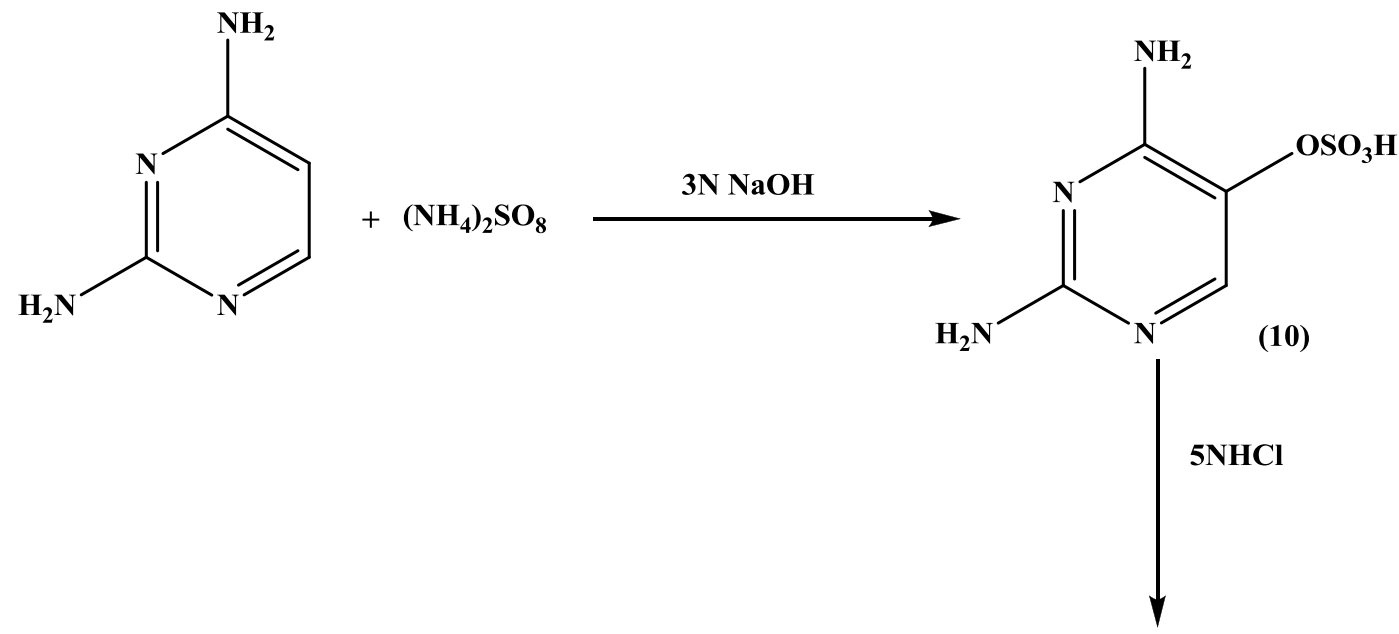<smiles>Nc1nc(N)c(O)c(Br)n1</smiles>

(12)

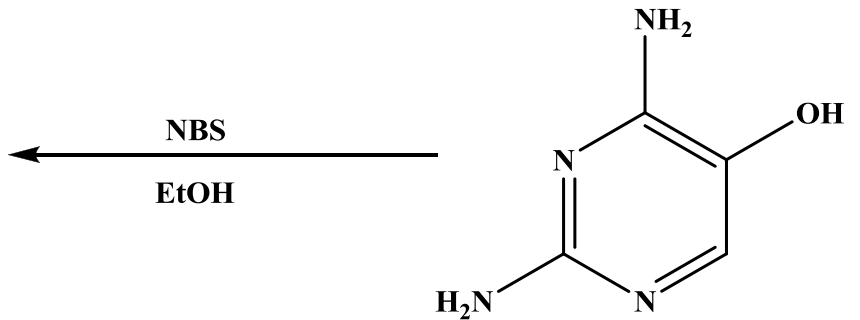

(11)<smiles>[R]c1ccc(N([R])CC(N)CS)cc1</smiles><smiles>[R]c1ccc(NCC2CSc3nc(N)nc(N)c3N2)cc1</smiles>

Scheme (2) 


\section{Se lection of PDB Structure}

The X-ray crystal structure of the protein (PDB ID: 3SRW) were retrieved from protein data bank based on good resolution and Ramachandran's plot analysis.

The crystal structures of S. aureus Dihydrofolate Reductase complexed with novel 7aryl-2,4-diaminoquinazolines have been determined to $1.70 \AA$ resolutions with sequence length of 167 base pairs (Fig 2).

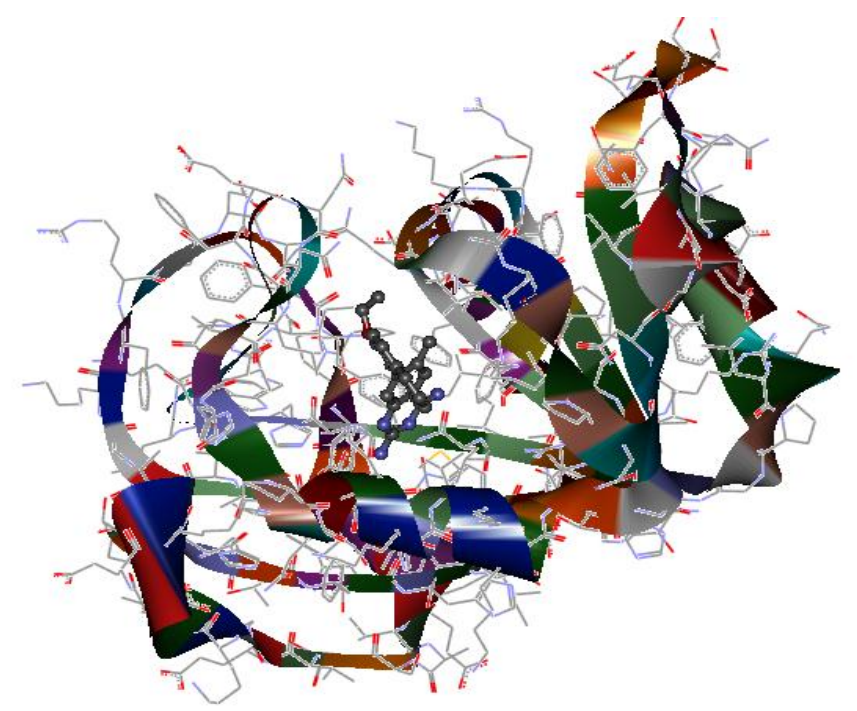

Fig 2: The structure of Dihydrofolate Reductase complexed with novel 7-aryl-2,4diaminoquinazolines

\section{DOCKING}

Docking of Synthetic ligands with 3SRW structure CDOCKER is a grid-based molecular docking method that employs CHARMm (Brooks et al., 1983). The receptor was held rigid while the ligand was allowed to flex during the refinement. By the search mentioned above, prior knowledge of the binding site had been acquired. Hence it was possible to specify the ligand placement in the active site using a binding site sphere with the radius of $10 \AA$ (Fig 3).

The CDOCKER interaction energy between the compounds and 3SRW (E-binding) was finally computed. From the docking analysis, insights into the interactions between the ligands and the receptor were gained, which facilitated the selection of top 6 poses which were saved for comparison and analysis.

Finally, the pose with the lowest CDOCKER energy was used for further study 


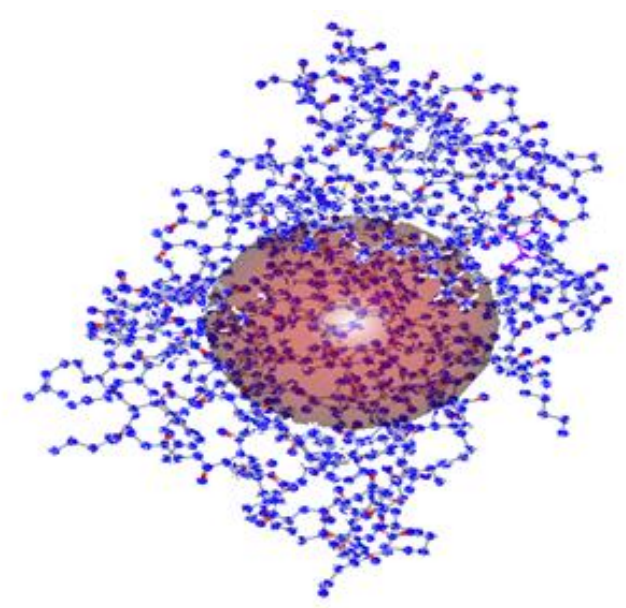

Fig 4: Defining of sphere around active site

Table 6: CDOCKER energy interaction of synthesized compounds (11.10 and 11.20) with (3SRW) at active site

\begin{tabular}{|l|l|l|l|}
\hline Comp. No. & $\begin{array}{l}\text { CDOCKER } \\
\text { INTERACTION } \\
\text { ENERGY }\end{array}$ & No.of Hydrogen Bonds \\
\hline 11.10 & Molecule & \\
\hline 11.10 & &
\end{tabular}




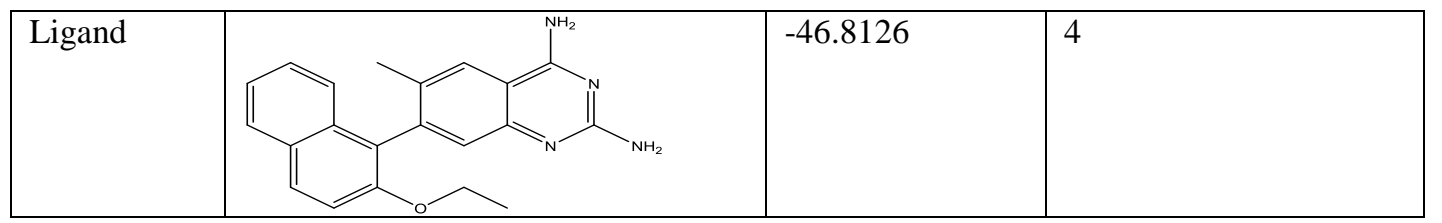

Ligand forms four hydrogen bounds with Asp. 19, Ser. 50, Thr. 47 and Thr. 122 and compound 11.10 form three hydrogen bonds with Asp. 19, Ser. 50, and extra hydrogen bond with glycine 96. But compound 11.20 form two hydrogen bonds with Ser. 50, and Thr. 47 similar to the ligand and the bound distance with the ligand is much more than with the

\section{Molecular docking studies and binding mode}

\section{a- Selection of PDB Structure}

The X-ray structures of the protein $3 S R W$ (S. aureus Dihydrofolate Reductase complexed with novel 7-aryl-2,4-diaminoquinazolines) were retrieved from protein data bank ${ }^{[1]}$ based on good resolution (1.7 $)$ and Ramachandran plot analysis.

\section{b- Ligand generation and Optimization}

Total 20 synthetic ligand compounds of Scheme-1, were drawn using ChemBioDraw 14.0 and saved in sdf format. The saved ligand compounds were later imported and minimized using Discovery Studio 2.5 after adding hydrogen bonds. The molecules thus obtained were saved in PDB format.

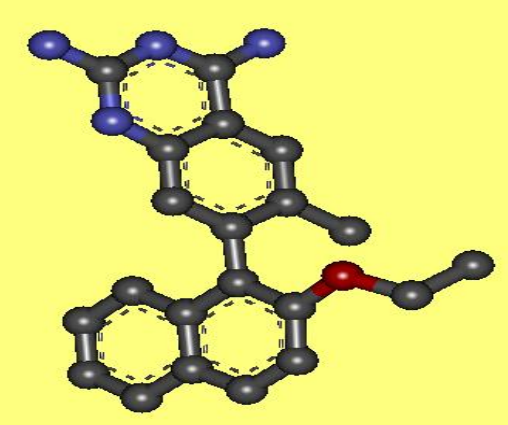

Fig 1: 7-(2-ethoxynaphthalen-1-yl)-6-methylquinazoline- 2,4-diamine ligand molecule

c- Molecular Docking using Discovery Studio 2.5

CDOCKER in Accelrys Discovery Studio uses a CHARMm-based molecular dynamics (MD) scheme to dock ligands into a receptor binding site. Random ligand conformations are generated using high-temperature MD. The conformations are then translated into the binding site. Candidate poses are then created using random rigidbody rotations followed by simulated annealing. A final minimization is then used to refine the ligand poses.( Hewth et al 1989) 


\section{RESULTS AND DISCUSSION}

Fig 4: Defining of sphere around active site

Table 6: CDOCKER energy interaction of synthesized compounds (11.10 and 11.20) with (3SRW) at active site

\begin{tabular}{|c|c|c|c|}
\hline $\begin{array}{l}\text { Comp. } \\
\text { No. }\end{array}$ & Molecule & $\begin{array}{l}\text { CDOCKER } \\
\text { INTERACTION } \\
\text { ENERGY }\end{array}$ & $\begin{array}{l}\text { No.of Hydrogen } \\
\text { Bonds }\end{array}$ \\
\hline 11.10 & & -49.1395 & 3 \\
\hline 11.10 & & -48.6565 & 3 \\
\hline 11.10 & & -48.5233 & 3 \\
\hline 11.20 & & -47.3358 & 2 \\
\hline Ligand & & -46.8126 & 4 \\
\hline
\end{tabular}

Ligand forms four hydrogen bounds with Asp. 19, Ser. 50, Thr. 47 and Thr. 122 and compound 11.10 form three hydrogen bonds with Asp. 19, Ser. 50, and extra hydrogen bond with glycine 96. But compound 11.20 form two hydrogen bonds with Ser. 50, and 
Thr. 47 similar to the ligand and the bound distance with the ligand is much more than with the screened compounds and this means that the binding of the screened compounds with the receptor is much more than the ligand

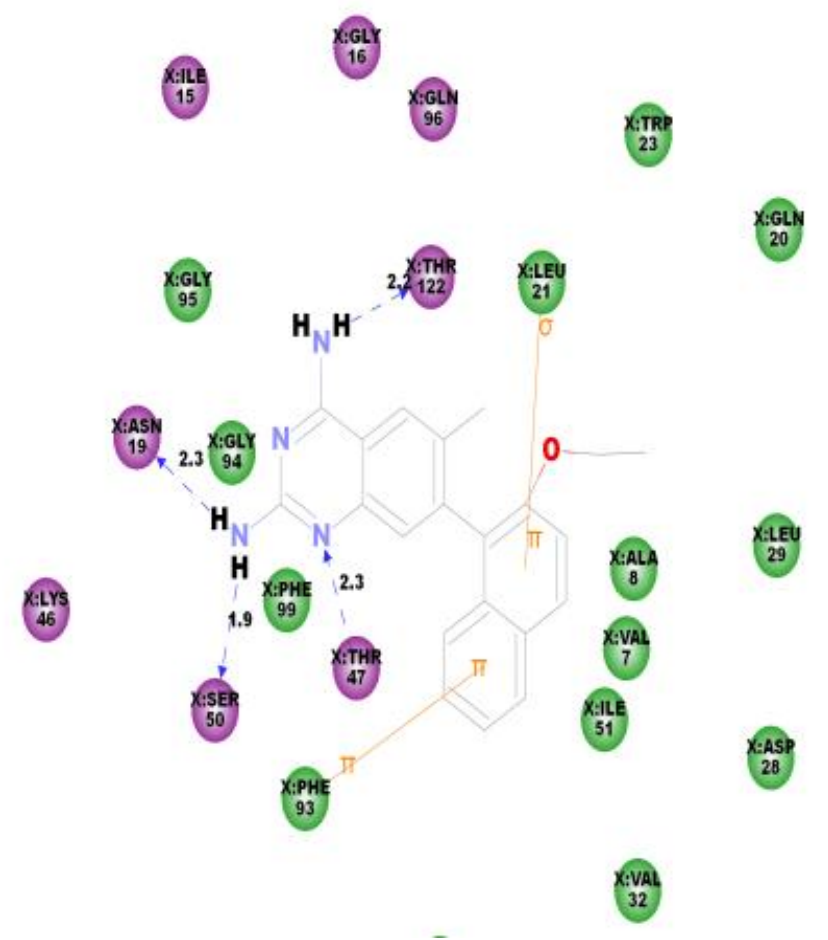

2D interaction diagram of ligand with (3srw) using Discovery Studio program with the essential amino acid residues at the binding site are tagged in circles.

(2)

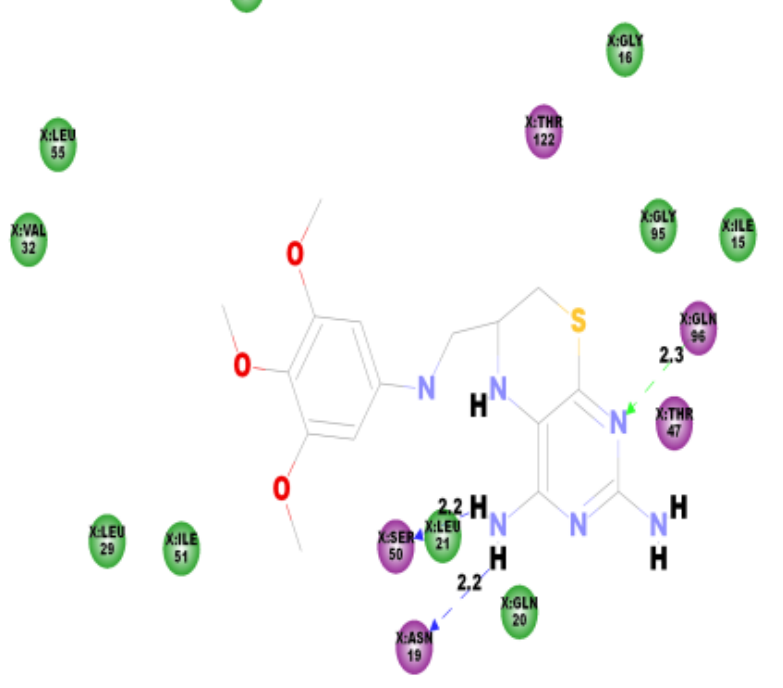

(다난

2D interaction diagram of compound (10) with (3srw) using Discovery Studio program with the essential amino acid residues at the binding site are tagged in circles. 


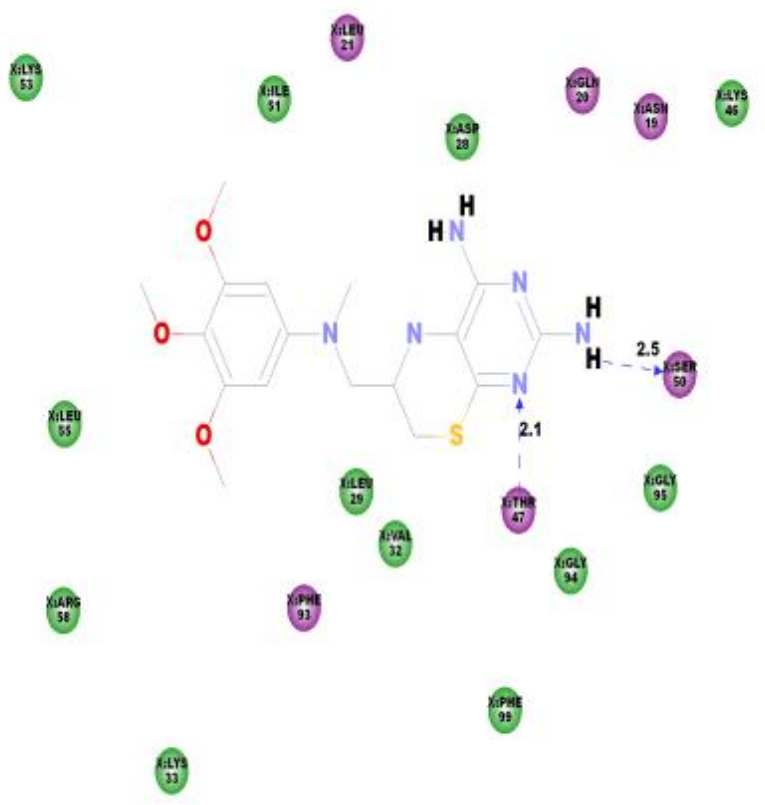

2D interaction diagram of compound (20) with (3srw) using Discovery Studio program with the essential amino acid residues at the binding site are tagged in circles.

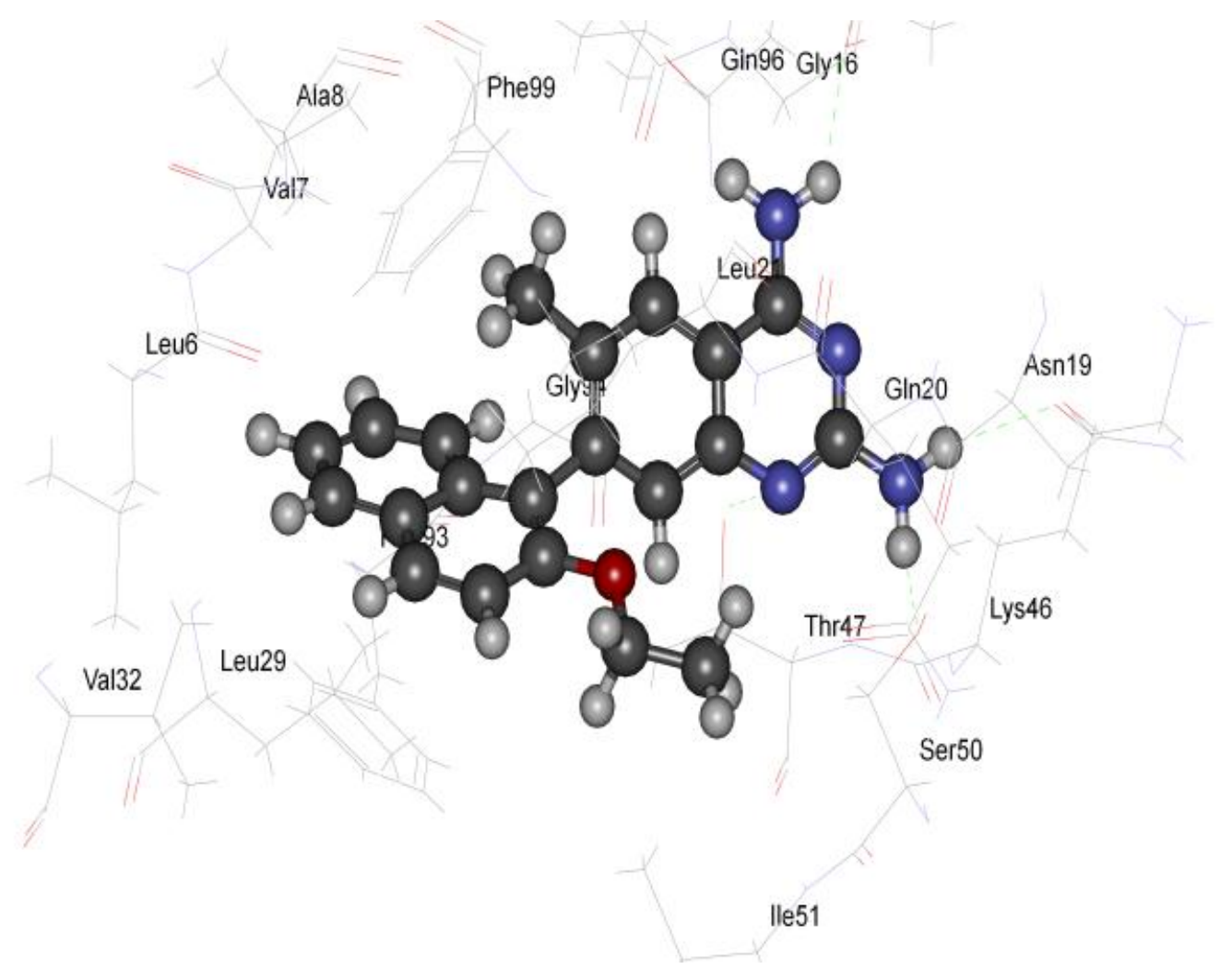


The proposed binding mode of ligand inside the active site of ( $3 \mathrm{srw})$ enzyme. The most important amino acids are shown together with their respective numbers

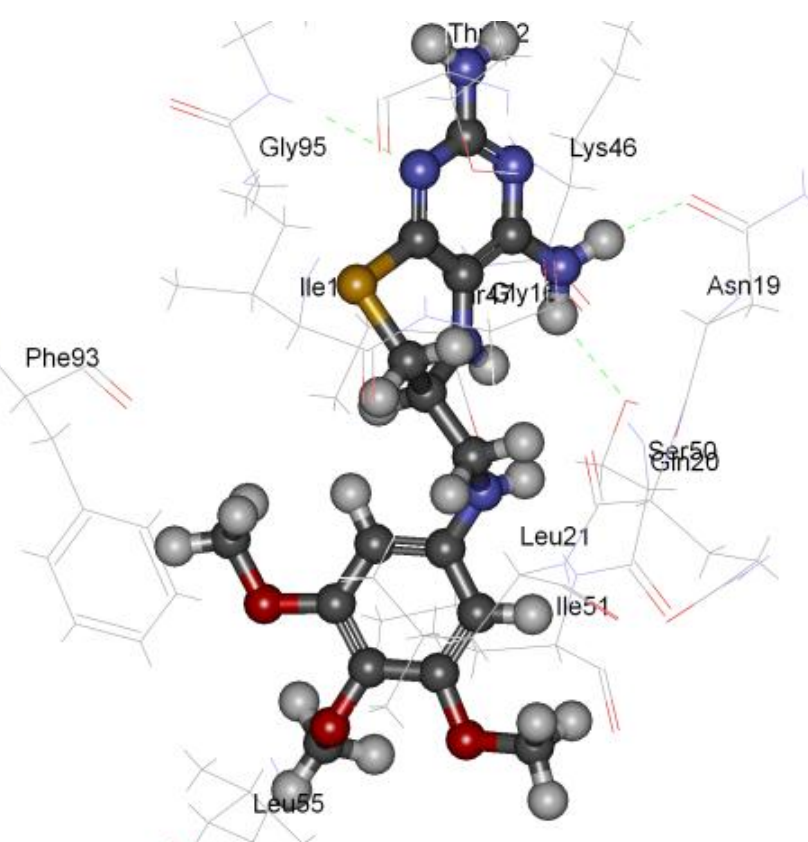

The proposed binding mode of compound (10), inside the active site of (3srw) enzyme.

The most important amino acids are shown together with their respective numbers

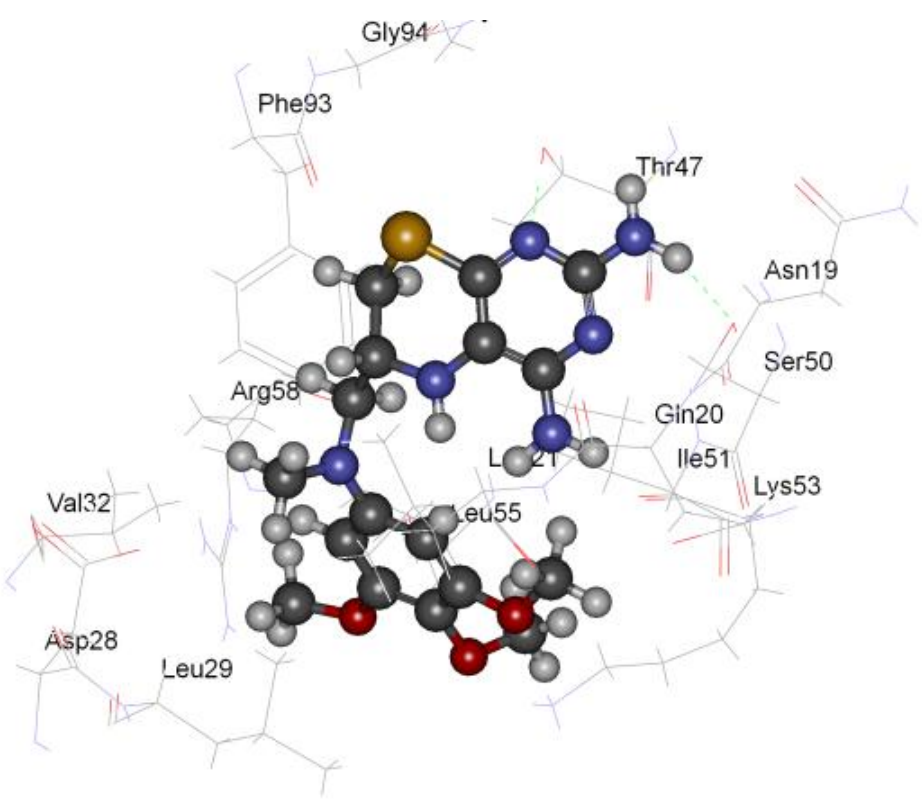

The proposed binding mode of compound (20), inside the active site of (3srw) enzyme. The most important amino acids are shown together with their respective numbers 


\section{REFERENCES}

Gangjee, A., Vasudevan, A., Queener, S.F., and Kisliuk, R.L., et al(1995) J. Med. Chem., , 38, 1778-1785.

Gangjee, A., Shi, J., Queener, S.F.,Barrows, L.R., and Kisliuk, R. L.et al(1993), J. Med. Chem., , 36, 3437-3443.

Gangjee, A., Zuh, Y., Queener, S.F., Francom, P., and Broom, A.D.et al(1996), J. Med. Chem., , 39, 1836-1845.

Gangjee, A., Zuh, Y., and Queener, S.F.et al(1998), J. Med. Chem., , 41, 4533-4541.

Gangjee, A., Vidwans, A.P., Vasudevan, A., Queener, S.F., Kisliuk, R.L., Cody, V.,

Gangjee, A.,(1997) Shi, J., and Queener, S.F., J. Med. Chem., , 40, 1930-1936.

Gangjee, A., Mavandadi, F., and Queener, S.F.et al(1997), J. Med. Chem., , 40, 1173-1177.

Gangjee, A., Vasudevan, A., and Queener, S.F.,et al( 1997) J. Med. Chem., , 40, 479485.

Gangjee, A., Devraj, R., and Queener, S.F.,et al(1997) J. Med. Chem., , 40, 470-478.

Gangjee, A., Mavandadi, F., Kisliuk, R.L., McGuire, J. J., and Queener, S.F.,et al(1996) J. Med. Chem., , 39, 4563-4568.

Gangjee, A., Vasudevan, A., Queener, S.F., and Kisliuk, R.L.et al(1996), J. Med. Chem., , 39, 1438-1446.

Gangjee, A., Zaveri, N., Kothare, M., and Queener, S.F. et al(1995), J. Med. Chem., , 38, 3660-3668.

Hewth, W. and Vincent, S., et al(1989) "Theory and Application of Microbiological Assay", Academic press INC, New York.

Li, R., Galitsky, N., Luft, et al(1998) J.R., and Pangborn, W., J. Med. Chem., , 41, 3426-3434.

Sakr, H.M.(2007) Az. J. Pharm. Sci., , 35, 112-118

Sakr, H. M.( 2000) Ph. D. Thesis, "Design and Synthesis of A Novel set of Potential Inhibitors of Thymidylate Synthesase for biological study,.

Wu, G. Robertson, D. H. Brooks, C. L. III, Vieth, M. et al(2003) Detailed Analysis of Grid-Based Molecular Docking: A Case Study of CDOCKER - A CHARMm-Based MD Docking Algorithm. J. Comp. Chem., 24, 1549. 


$$
\text { الملخص العربى }
$$

تشييد بعض مركبات البيرميدوثيازين بهدف دراستها كمضاده للميكروبات

$$
\text { حلمى مصطفى صقر }
$$

قسم الكيمياء الصيدليهـ كليه الصيدله (بنين)- جامعه الأزهر - مدينه نصر - القاهره

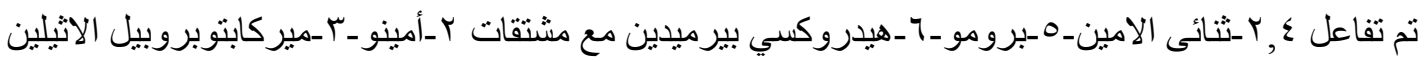

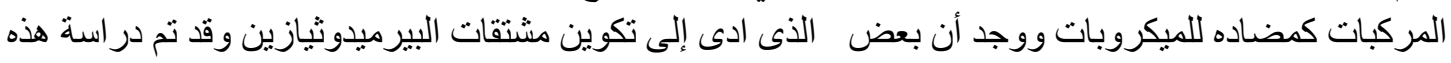
هذه المركبات لها فاعليه كمضاده للميكروبات بمقارنتها بالكلور امفينكول. 